
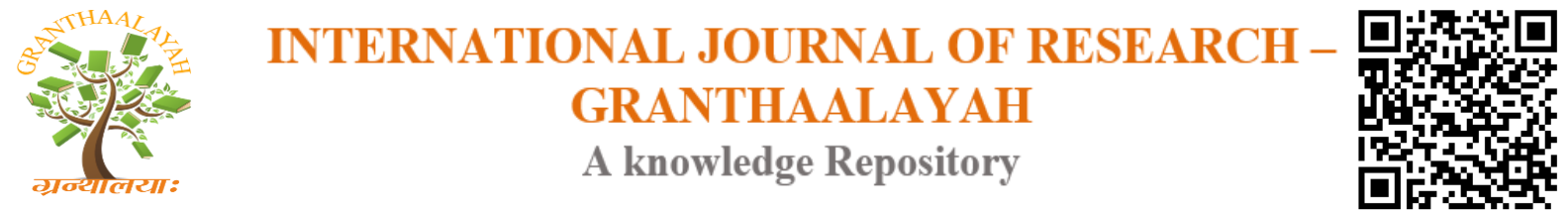

Science

\title{
MATHEMATICAL ANALYSIS OF SENSITIVE PARAMETERS ON THE DYNAMICAL TRANSMISSION OF EBOLA HEMORRHAGIC FEVER
}

\author{
S .O Adewale ${ }^{* 1}$, G.A Adeniran ${ }^{2}$, I .A Olopade ${ }^{3}$, I.T Mohammed ${ }^{4}$ \\ ${ }^{* 1,2}$ Departmental of P/AMathematics, Ladoke Akintola University of Technology, LAUTECH \\ Ogbomoso. NIGERIA \\ ${ }^{3}$ Departmental of Mathematics and Computer Science,Elizade University, Ilara-Mokin. \\ NIGERIA \\ ${ }^{4}$ Department of Mathematics and Stastitics Osun State Polytechnic Iree, NIGERIA
}

DOI: https://doi.org/10.29121/granthaalayah.v4.i10.2016.2484

\section{ABSTRACT}

A four (4) compartmental model of $(S, E, I, I)$ were presented to have better understanding of parameters that influence the dynamical spread of Ebola in a population. The model is analyzed for all the parameters responsible for the dynamical spread of the disease in order to find the most sensitive parameters that need to be given attention.

The stability of the model was analyzed for the existence of disease free and endemic equilibrium points. Basic Reproduction Number () was obtained using next generation matrix method (NGM), and it is shown that the disease free equilibrium point is locally asymptotically stable whenever the basic reproduction number is less than unity i.e ( ) and unstable whenever the basic reproduction number is greater than unity ( ).The relative sensitivity indices of the model with respect to each parameter in the basic reproduction number is calculated in order to find the most sensitive parameter which the medical practitioners and policy health makers should work on in order to reduce the spread of Ebola in the population. The result shows that effective contact rate and fraction of individuals with low immunity are the most sensitive parameters in the reproduction number. Therefore, effort should be put in place so that the basic reproduction number should not be greater unity so as to prevent the endemic situation.

Keywords:

Ebola; Reproduction Number; Stability; Critical Point; Sensitivity; Simulation.

Cite This Article: S .O Adewale, G.A Adeniran, I .A Olopade and I.T Mohammed, "MATHEMATICAL ANALYSIS OF SENSITIVE PARAMETERS ON THE DYNAMICAL TRANSMISSION OF EBOLA HEMORRHAGIC FEVER" International Journal of Research Granthaalayah, Vol. 4, No. 10 (2016): 21-33. 


\section{INTRODUCTION}

Ebola hemorrhagic fever is an infectious and deadly disease that is majorly caused by infection with one of the Ebola virus species which occurred in both humans and non-humans primates (Chimpanzees, Monkeys and Gorillas). This deadly disease causes an acute and serious illness and was discovered around 1976 near the Ebola River in the Democratic Republic of the Congo, where it takes its name. [8, 14, 15]. It was recorded that 12 outbreaks of Ebola virus appeared in Congo, Sudan, Gabon, and Uganda around September 14, 2003. [7, 14]. In those particular areas, two different strains of Ebola virus (Ebola-Zaire and the Ebola-Sudan) were discovered and reported. Despite the efforts put in place so far by researchers, the reservoir of Ebola virus has not been detected $[3,11]$.

Ebola Virus is transmitted into human population through contacts with the blood, organs or the bodily fluids and secretions of an infected animals like chimpanzees, fruit bats, gorilla, forest antelope and porcupines found dead in the rainforest $[14,15,16]$. This virus is a member of the family Filoviridae, which are the family of viruses that cause severe hemorrhagic fever (characterized by high fever, internal bleeding, hypertension and shock) in humans. There are five recognized strains of Ebola virus species, four of which are known to cause disease in human population which are Zaire ebolavirus, Sudan ebolavirus, Tai Forest ebolavirus (Known as Cote d'Ivoire ebolavirus) and Bundibugyo virus. The fifth ebola virus known as Restoen Virus causes disease in non-human Primates .When an individual comes in contact, and gets infected by the virus, the virus develops in the body within a period of 2 to 21 days $[8,17]$.

Individuals that are infected with ebola virus develops the following symptoms: diarrhea, vomiting, rash, symptoms of impaired kidney and liver functions, and in some cases, both internal and external bleeding (e.g blood in the stools). Individuals that are infected receive little medical attention due to the fact that the vaccine to curb its spread is yet to be developed [15, 16]. Furthermore, bats (known as fruit bats) have been detected as the major carrier of the disease $[4,10]$.

As a result of competition for food (fruit) in the dry season, contact between bats and gorilla occurs and this has not really revealed the way by which other animals like duikers, become infected. Although duikers might come in contact with the virus by consuming fallen fruits [13]. Many studies have been carried out to investigate the transmission dynamics of Ebola disease. Adewale et al. [1] Analyzed the effects of isolation on Ebola transmission dynamics. It was concluded in their study that if the isolation rate of Exposed and Infected individuals is high and the effective contact rate $(\beta)$ ranges between $(0.113-0.9)$, the disease will be eradicated in the community. Chowell et al. [5] studied the basic reproduction number of Ebola and the effects of public health measures: the cases of Congo and Uganda. They presented a mathematical model to study the course of the outbreaks via an SEIR (Susceptible-exposed-infected-removed) epidemic model that includes a smooth transition in the transmission rate after control interventions are put in place. Amira R and Delfim F.M [2] studied a simple Mathematical model that describes the 2015 Ebola outbreak in Liberia. They used numerical simulations and available data provided by the World health Organization to validate the obtained mathematical model. They also developed a new mathematical model including vaccination of individuals over time. They applied optimal control to study the impact of vaccination on the spread of ebola virus. 
In this present work, a four (4) compartmental model of $\left(\mathrm{S}, \mathrm{L}, \mathrm{I}_{D}, \mathrm{I}_{U}\right)$ was presented to check the sensitive parameters on the basic reproduction number in order to have better understanding of parameters that influence the dynamical spread of Ebola.

\section{MATHEMATICAL MODEL FORMULATION}

The model considered four (4) compartmental models to have better understanding of parameters that influence the dynamical spread of Ebola in a community. The model comprises of Susceptible Individuals $\mathrm{S}(\mathrm{t})$, Latently Individuals $\mathrm{L}(\mathrm{t})$, Infected detected Individuals $I_{D}(t)$, Infected undetected Individuals $I_{U}(t)$ so that

Population at time $\mathrm{t}$ denoted by $\mathrm{N}(\mathrm{t})$ is sub-divided into 4 compartments of Susceptible $\mathrm{S}(\mathrm{t})$, Latent $\mathrm{L}(\mathrm{t})$, Infected detected $I_{D}(t)$ and Infected Undetected $I_{U}(t)$ individuals. Where

$$
N(t)=S(t)+L(t)+I_{D}(t)+I_{U}(t)
$$

The susceptible population is increased by the recruitment of people (either by birth or immigration) into the population at the rate $\pi$, (all recruited individuals are assumed to be susceptible), also the susceptible population decreases by infection which can be acquired following effective contact with infectious individuals only at a rate $\lambda$ given by

$$
\lambda=\frac{\beta\left(L+\eta_{1} I_{D}+\eta_{2} I_{U}\right)}{N}
$$

In (2) $\beta$ represents the effective contact rate (i.e contact capable of leading to Ebola infection). $\eta_{1}$ and $\eta_{2}$ are the modification parameters that compares the transmissibility of disease.

Thus the rate of change of the susceptible population is given by

$$
\frac{d S}{d t}=\pi-\lambda S-\mu S
$$

A fraction $\varepsilon$ of new infected individuals with low immunity move to the latent class (L), while the remaining fraction $(1-\varepsilon)$ move to the infected detected class $\left(\mathrm{I}_{D}\right)$. The population is further decreased by progression rate $\kappa$ and the natural death of individuals at the rate $\mu$. Thus,

$$
\frac{d L}{d t}=\varepsilon \lambda S-(\kappa+\mu) L
$$

The population of infected detected individuals is increased by the new infection due to low immunity in their body at the rate $(1-\varepsilon)$ and by endogenous reactivation from latent class to infected detected at the rate $\omega_{1} \kappa$, the detection of infected undetected individual at the rate $\gamma$ and finally decreased by the natural death of individual at the rate $\mu$ and the disease induced death at the rate $\delta_{D}$.

Thus,

$$
\frac{d I_{D}}{d t}=(1-\varepsilon) \lambda S+\omega_{1} \kappa L-\left(\mu+\delta_{D}\right) I_{D}+\gamma I_{U}
$$

In the model, we also assumed that some people have been infected but not detected. These individuals are increased by endogenous reactivation of symptoms by latent individuals at the rate $\left(1-\omega_{1}\right) \kappa$ and finally reduced due to the detection of infected undetected individuals at the rate $\gamma$, natural death of individual at the rate $\mu$ and the disease induced death at the rate $\delta_{U}$. Thus, 
$\frac{d I_{U}}{d t}=\left(1-\omega_{1}\right) \kappa L-\left(\gamma+\mu+\delta_{U}\right) I_{U}$

Thus in summary, the Ebola dynamic transmission model is given by the following system of non-linear differential equations,

$$
\left.\begin{array}{l}
\frac{d S}{d t}=\pi-\lambda S-\mu S \\
\frac{d L}{d t}=\varepsilon \lambda S-(\kappa+\mu) L \\
\frac{d I_{D}}{d t}=(1-\varepsilon) \lambda S+\omega_{1} \kappa L-\left(\mu+\delta_{D}\right)+\gamma I_{U} \\
\frac{d I_{U}}{d t}=\left(1-\omega_{1}\right) \kappa L-\left(\gamma+\mu+\delta_{U}\right) I_{U}
\end{array}\right\}
$$

Table 1: Description of Variables and Parameters

\begin{tabular}{|l|l|}
\hline Parameters & Definitions \\
\hline $\mathrm{S}$ & Susceptible Individuals \\
\hline $\mathrm{L}$ & Latently Individuals \\
\hline$I_{D}$ & Infected detected Individuals \\
\hline$I_{U}$ & Infected Undetected Individuals \\
\hline$\lambda$ & Force of Infection \\
\hline$\beta$ & Effective Contact rate \\
\hline$\pi$ & Recruitment rate into Susceptible \\
\hline$\varepsilon$ & Fraction of Individuals with Low Immunity \\
\hline$\kappa$ & Progression rate \\
\hline$\mu$ & Natural death rate \\
\hline$\delta$ & Disease Induced Death rate \\
\hline$\gamma$ & Detection rate \\
\hline$\omega$ & Endogenous Reactivation rate \\
\hline$\eta_{1}, \eta_{2}$ & Modification Parameters \\
\hline
\end{tabular}

\section{MATHEMATICAL ANALYSIS}

\section{Lemma 1:}

The close set $D=\left\{\left(S, L, I_{D}, I_{U}\right) \in R_{+}^{4}: N \leq \frac{\pi}{\mu}\right\}$ is positively-invariant and attracting with respect to model (7)

Proof 1: Consider the biologically-feasible region.

$D=\left\{\left(S, L, I_{D}, I_{U}\right) \in R_{+}^{4}: N \leq \frac{\pi}{\mu}\right\}$. We shall show that $\mathrm{D}$ is positive invariance (i.e all solutions remain in $\mathrm{D}$ for all time $\mathrm{t}>0$ ). The rate of change of the total population obtained by adding all the equations in the model above is given by 
$\frac{d N}{d t}=\pi-\mu N-\delta_{D} I_{D}-\delta_{U} I_{U}$

It follows that $\frac{d N}{d t}<0$ whenever $N>\frac{\pi}{\mu}$. Note that $\frac{d N}{d t}$ is bounded by $\pi-\mu N$ and a standard comparism theorem [12] can be used to show that $N(t) \leq N(0) e^{-\mu t}+\frac{\pi}{\mu}\left(1-e^{-\mu t}\right)$. In particular $N(t) \leq \frac{\pi}{\mu}$, if $N(0) \leq \frac{\pi}{\mu}$, Therefore all solution of the model with initial conditions in $\mathrm{D}$ is positively-invariant and attracting. In this region the model can be considered as been epidemiologically and mathematically well-posed.

\subsection{STABILITY OF THE DISEASE FREE EQUILIBRIUM (DFE)}

Disease free equilibrium

For critical points, we set

$$
\frac{d S}{d t}=\frac{d L}{d t}=\frac{d I_{D}}{d t}=\frac{d I_{U}}{d t}=0
$$

At disease free equilibrium, it is assumed that there is no infection, so we set

$L=I_{D}=I_{U}=0$

Disease free equilibrium of Ebola is given by

$\varepsilon_{0}=\left(\frac{\pi}{\mu}, 0,0,0\right)$

\subsection{DERIVATION OF BASIC REPRODUCTION NUMBER $\left(R_{0}\right)$}

The basic reproduction number of the model (7) is calculated by using next generation matrix [9]. Using their approach, we have

$$
\begin{aligned}
F_{i} & =\left(\begin{array}{l}
F_{1} \\
F_{2} \\
F_{3}
\end{array}\right)=\left(\begin{array}{l}
\varepsilon \lambda S \\
(1-\varepsilon) \lambda S \\
0
\end{array}\right) \\
V_{i} & =\left(\begin{array}{l}
-(\kappa+\mu) L \\
\omega_{1} \kappa L-\left(\mu+\delta_{D}\right) I_{D}+\gamma I_{U} \\
\left(1-\omega_{1}\right) \kappa L-\left(\gamma+\mu+\delta_{U}\right) I_{U}
\end{array}\right)
\end{aligned}
$$

Where

$$
F=\left[\begin{array}{ccc}
\beta \varepsilon & \beta \varepsilon \eta_{2} & \beta \varepsilon \eta_{3} \\
(1-\varepsilon) \beta & (1-\varepsilon) \beta \eta_{2} & (1-\varepsilon) \beta \eta_{3} \\
0 & 0 & 0
\end{array}\right]
$$

and 


$$
V=\left[\begin{array}{ccc}
k_{1} & 0 & 0 \\
-\omega_{1} \kappa & -k_{2} & -\gamma \\
-k_{3} & 0 & -k_{4}
\end{array}\right]
$$

The reproduction number is the dominant eigen values of $F \times V^{-1}$. Thus,

$$
R_{0}=\frac{\beta\left(-\eta_{1} k_{1} k_{4} \varepsilon+\eta_{1} k_{1} k_{4}+\varepsilon \kappa k_{4} \eta_{2} \omega_{1}+\gamma \varepsilon \eta_{1} k_{3}+\varepsilon \eta_{2} k_{2} k_{3}+\varepsilon k_{4} k_{2}\right)}{k_{1} k_{2} k_{4}}
$$

Where $k_{1}=\kappa+\mu, k_{2}=\mu+\delta_{D}, k_{3}=\left(1-\omega_{1}\right) \kappa$ and $k_{4}=\gamma+\mu+\delta_{U}$

\subsection{LOCAL STABILITY OF DISEASE FREE EQUILIBRIUM (DFE)}

Theorem 1: The disease free equilibrium of the system (7) is locally asymptotically stable (LAS) if $R_{0}<1$ and unstable if $R_{0}>1$

Proof: To determine the local stability of $E_{0}$, the following matrix is computed corresponding to equilibrium point $E_{0}$. Considering the stability of the disease free equilibrium at the critical point $(0,0,0,0)$. Equation (6), we have

$$
J_{E}=\left(\begin{array}{cccc}
-\mu & 0 & 0 & 0 \\
0 & -k_{1} & 0 & 0 \\
0 & \omega_{1} \kappa & -k_{3} & 0 \\
0 & -k_{2} & 0 & -k_{4}
\end{array}\right)
$$

Then the characteristics equations are obtained as $\left|J_{E}-\lambda I=0\right|$ (where I is $4 * 4$ identity matrix). Where,

$$
\lambda I=\left(\begin{array}{llll}
\lambda & 0 & 0 & 0 \\
0 & \lambda & 0 & 0 \\
0 & 0 & \lambda & 0 \\
0 & 0 & 0 & \lambda
\end{array}\right)
$$

Hence $\left|J_{E}-\lambda I=0\right|$ implies that

From equation (11), clearly $\lambda_{1}=-\mu, \lambda_{2}=-k_{1}, \lambda_{3}=-k_{3}, \lambda_{4}=-k_{4}$

Hence, the corresponding eigen values of equation (7) are;

$\lambda_{1}=-\mu, \lambda_{2}=-(\kappa+\mu), \lambda_{3}=-\left(\mu+\delta_{D}\right), \lambda_{4}=-\left(\gamma+\mu+\delta_{U}\right)$

Since all the eigen values are real, negative and distinct, hence disease free equilibrium of the system (7) is locally asymptotically stable (LAS).

\subsection{GLOBAL STABILITY OF DISEASE FREE EQUILIBRIUM}

Here, the global stability (GAS) property of the DFE of the system (7) shall be explored.

Theorem 2: The disease free equilibrium of the system (7) is globally asymptotically stable whenever $R_{0}<1$ and unstable if $R_{0}>1$. 
Proof: It follows that $S=N^{*}-L-I_{D}-I_{U}$ at steady state. The proof is based on using the comparison theorem [12] to prove the global stability. The rate of change of the variables representing the infected component of the system can be written as follows.

$$
\begin{aligned}
& \frac{d E}{d t}=\varepsilon \lambda\left(N^{*}-L-I_{D}-I_{U}\right)-k_{1} L \\
& \frac{d I_{D}}{d t}=(1-\varepsilon) \lambda\left(N^{*}-L-I_{D}-I_{U}\right)+\omega_{1} \kappa-k_{2} I_{D}+\gamma I_{U} \\
& \frac{d I_{U}}{d t}=k_{3} L-k_{4} I_{U} \\
& D^{*}=\left\{\left(E, I_{D}, I_{U}\right) \in R_{+}^{3}: L+I_{D}+I_{U} \leq N^{*}\right\}
\end{aligned}
$$

For the model (6), the associated reproduction number, denoted by $R_{0}$ where

$$
R_{0}=\frac{\beta\left(-\eta_{1} k_{1} k_{4} \varepsilon+\eta_{1} k_{1} k_{4}+\varepsilon \kappa k_{4} \eta_{1} \omega_{1}+\gamma \varepsilon \eta_{1} k_{3}+\varepsilon \eta_{2} k_{2} k_{3}+\varepsilon k_{4} k_{2}\right)}{k_{1} k_{2} k_{4}}
$$

The DFE of the model (6) is GAS in $\mathrm{D}^{*}$ if $R_{0}<1$.

Using comparison method, we have

$$
\begin{aligned}
& \left(\begin{array}{l}
\frac{d L}{d t} \\
\frac{d I_{D}}{d t} \\
\frac{d I_{U}}{d t}
\end{array}\right)=(F-V)\left(\begin{array}{l}
L \\
I_{D} \\
I_{U}
\end{array}\right)-F_{i}\left(\begin{array}{l}
L \\
I_{D} \\
I_{U}
\end{array}\right) \\
& {\left[\begin{array}{ccc}
\left(\beta \varepsilon-k_{1}\right)-\lambda & \beta \varepsilon \eta_{1} & \beta \varepsilon \eta_{2} \\
(1-\varepsilon) \beta+\omega_{1} \kappa & \left((1-\varepsilon) \beta \eta_{1}-k_{2}\right)-\lambda & (1-\varepsilon) \beta \eta_{2}+\gamma \\
k_{3} & 0 & -k_{4}-\lambda
\end{array}\right]=0}
\end{aligned}
$$

From equation (14), we have

$$
\begin{aligned}
& \lambda^{3}-\left(-\varepsilon \beta \eta_{1}+\varepsilon \beta+\beta \eta_{1}-k_{1}-k_{2}-k_{4}\right) \lambda^{2}-\left(k_{3} \varepsilon \beta \eta_{1}-\varepsilon \beta k_{4} \eta_{1}+\varepsilon \beta k_{4}+\beta k_{4} \eta_{1}-k_{1} k_{4}-k_{2} k_{4}\right. \\
& \left.+\varepsilon \beta \kappa \eta_{1} \omega_{1}-\varepsilon \beta k_{1} \eta_{1}+\varepsilon \beta k_{2}+\beta k_{1} \eta_{1}-k_{1} k_{2}\right) \lambda-\gamma \varepsilon \beta \eta_{1} k_{3}-\varepsilon \beta k_{2} \eta_{2} k_{3}+\varepsilon \beta k_{1} k_{4} \eta_{1}-\varepsilon \beta \kappa k_{4} \eta_{1} \omega_{1} \\
& -\varepsilon \beta k_{2} k_{4}-\beta k_{1} k_{4} \eta_{1}+k_{1} k_{2} k_{4}
\end{aligned}
$$

Thus, from equation (15), the characteristics equation is given by

$$
\lambda^{3}+A_{3} \lambda^{2}+A_{2} \lambda+A_{1}
$$

Where

$$
\begin{aligned}
& A_{3}=-\left(-\varepsilon \beta \eta_{1}+\varepsilon \beta+\beta \eta_{1}-k_{1}-k_{2}-k_{4}\right) \\
& A_{2}=\left(k_{3} \varepsilon \beta \eta_{2}-\varepsilon \beta k_{4} \eta_{1}+\varepsilon \beta k_{4}+\beta k_{4} \eta_{1}-k_{1} k_{4}-k_{2} k_{4}\right. \\
& \left.+\varepsilon \beta \kappa \eta_{1} \omega_{1}-\varepsilon \beta k_{1} \eta_{1}+\varepsilon \beta k_{2}+\beta k_{1} \eta_{1}-k_{1} k_{2}\right) \\
& A_{1}=-\gamma \varepsilon \beta \eta_{1} k_{3}-\varepsilon \beta k_{2} \eta_{1} k_{3}+\varepsilon \beta k_{1} k_{4} \eta_{1}-\varepsilon \beta \kappa k_{4} \eta_{1} \omega_{1} \\
& -\varepsilon \beta k_{2} k_{4}-\beta k_{1} k_{4} \eta_{1}+k_{1} k_{2} k_{4}
\end{aligned}
$$

According to Routh Hurwitz Criteria of Order three (3), $n=3: A_{1}>0, A_{3}>0$, and $A_{1} A_{2}>A_{3}$. 
Therefore for $A_{1}>0$ we have

$\frac{\beta\left(-\eta_{1} k_{1} k_{4} \varepsilon+\eta_{1} k_{1} k_{4}+\varepsilon \kappa k_{4} \eta_{1} \omega_{1}+\gamma \varepsilon \eta_{1} k_{3}+\varepsilon \eta_{1} k_{2} k_{3}+\varepsilon k_{4} k_{2}\right)}{k_{1} k_{2} k_{4}}<1$,

Implying that

$R_{0}<1$

Hence, we have established that the disease free is globally asymptotically stable whenever $R_{0}<$ 1 and unstable if $R_{0}>1$.

\subsection{EXISTENCE OF ENDEMIC EQUILIBRIUM POINT OF THE MODEL}

Let $\varepsilon_{1}=\left(S^{* *}, L^{* *}, I_{D}^{* *}, I_{U}^{* * *}\right)$ represents any arbitrary endemic equilibrium of the model at steadystate gives

$$
\begin{aligned}
& S^{* *}=\frac{\pi}{\lambda^{* *}+\mu} \\
& L^{* *}=\frac{\varepsilon \lambda^{* *} S^{* * *}}{k_{1}} \\
& I_{D}^{* *}=\frac{(1-\varepsilon) \lambda^{* *} S^{* *}+\omega_{1} \kappa L^{* *}+\gamma I_{U}^{* *}}{k_{2}} \\
& I_{U}^{* *}=\frac{\left(1-\omega_{1}\right) \kappa L^{* *}}{k_{3}}
\end{aligned}
$$

Where

$$
\begin{aligned}
& k_{1}=(\kappa+\mu) \\
& k_{2}=\left(\mu+\delta_{D}\right) \\
& k_{3}=\left(\gamma+\mu+\delta_{U}\right)
\end{aligned}
$$

The expression for $\lambda$ at the endemic steady-state, denoted by $\lambda^{* *}$ is given by

$$
\lambda^{* *}=\beta\left[\frac{L^{* *}+\eta_{1} I_{D}^{* *}+\eta_{2} I_{U}^{* *}}{N^{* *}}\right]
$$

For computational convenience, we write $L^{* *}, I_{D}^{* * *}$ and $I_{U}^{* *}$ in terms of

$L^{* *}=\frac{\varepsilon \lambda^{* *} S^{* *}}{k_{1}}=P_{0} \lambda^{* *} S^{* *}$

$I_{U}^{* *}=\frac{\left(1-\omega_{1}\right) \kappa L^{* *}}{k_{3}}=P_{1} \lambda^{* * *} S^{* *}$

$I_{D}^{* *}=\frac{(1-\varepsilon) \lambda^{* *} S^{* *}}{k_{2}}+\frac{\omega_{1} \kappa \lambda^{* *} S^{* *}}{k_{1} k_{2}}+\frac{\gamma\left(1-\omega_{1}\right) \kappa \varepsilon \lambda^{* *} S^{* *}}{k_{2} k_{3}}=P_{2} \lambda^{* *} S^{* *}$

Where,

$$
P_{0}=\frac{\varepsilon}{k_{1}}
$$




$$
\begin{aligned}
& P_{1}=\frac{\left(1-\omega_{1}\right) \kappa \varepsilon}{k_{3}} \\
& P_{2}=\frac{1-\varepsilon}{k_{2}}+\frac{\omega_{1} \kappa}{k_{1} k_{2}}+\frac{\gamma\left(1-\omega_{1}\right) \kappa \varepsilon}{k_{2} k_{3}}
\end{aligned}
$$

Substituting 21, 22 and 23 into 20 we have

$$
\begin{aligned}
& \lambda^{* *}\left[S^{* *}+L^{* *}+I_{D}^{* *}+I_{U}^{* *}\right]=\beta\left[L^{* *}+\eta_{1} I_{D}^{* *}+\eta_{2} I_{U}^{* *}\right] \\
& \lambda^{* *}\left[S^{* *}+\frac{\varepsilon \lambda^{* *} S^{* *}}{k_{1}}+P_{1} \lambda^{* * *} S^{* *}+P_{2} \lambda^{* *} S^{* *}\right]=\beta \lambda^{* *} S^{* *}\left[P_{0}+\eta_{1} P_{1}+\eta_{2} P_{2}\right]
\end{aligned}
$$

Dividing each term in (24) the above by $\lambda^{* *} S^{* *}$ gives

$$
\begin{aligned}
& 1+P_{4} \lambda^{* *}=\quad \beta\left[P_{0}+\eta_{1} P_{1}+\eta_{2} P_{2}\right] . \\
& \quad \text { Where } \\
& p_{4}=\frac{\varepsilon}{k_{11}}+p_{1}+p_{2} \geq 0 .
\end{aligned}
$$

So that,

$$
1+P_{4} \lambda^{* *}=\beta\left[\frac{\varepsilon}{k_{1}}+\frac{\eta_{1}\left(1-\omega_{1}\right) \kappa \varepsilon}{k_{3}}+\eta_{2}\left\{\frac{1-\varepsilon}{k_{2}}+\frac{\omega_{1} \kappa}{k_{1} k_{2}}+\frac{\gamma\left(1-\omega_{1}\right) \kappa \varepsilon}{k_{2} k_{3}}\right\}\right]
$$

Therefore, $1+P_{4} \lambda^{* *}=R_{0}$

So that $\lambda^{* *}=\frac{R_{0}-1}{P_{4}}>0$, Where $R_{0}>1$.

\subsection{SENSITIVITY OF THE MODEL}

It is necessary to determine how sensitive the threshold quantity basic reproduction number is with respect to its parameters, this will help to understand which of the parameters causes reduction in $R_{O}$ and parameters that increases $R_{O}$ and these parameters must be given attention in order to have most effective control of the disease. This analysis will help to know how important each parameter is to disease transmission. We compute the normalized forward sensitivity index of the reproduction number with respect to its parameters.

Definition: If a variable ' $\mathrm{k}$ ' depends differentiably on a parameter ' $\mathrm{q}$ ', then, the normalized forward sensitivity index of ' $\mathrm{k}$ ' with respect to with respect to ' $\mathrm{q}$ ' is denoted by $X_{k}=\frac{k}{q} \frac{\partial q}{\partial k}$

As we have explicit relation for $R_{0}$, we generated an analytical expression for the sensitivity of $R_{0}$ as

$X_{q}^{R_{0}}=\frac{d R_{0}}{d q} \times \frac{q}{R_{0}}$

Sensitivity analysis for each parameter involved in $R_{0}$ is therefore calculated and show in the table 2 
Table 2: Values of Numerical Sensitivity of the model

\begin{tabular}{|c|c|}
\hline Parameters & Sensitivity Values \\
\hline$\gamma$ & $-7.0128434 \times 10^{-11}$ \\
\hline$\beta$ & 0.999910 \\
\hline$\mu$ & -0.032624 \\
\hline$\kappa$ & 0.947708 \\
\hline$\omega_{1}$ & 0.000000 \\
\hline$\delta_{U}$ & -0.015554 \\
\hline$\delta_{D}$ & -0.004113 \\
\hline$\varepsilon$ & 0.979299 \\
\hline$\eta_{2}$ & 0.004201 \\
\hline$\eta_{3}$ & 0.015886 \\
\hline
\end{tabular}

\section{NUMERICAL SIMULATION}

The model was solved numerically using Runge-kutta of order four (4). In order to verify the effects of contact rate and low immunity rate in the dynamical spread of ebola, the following set of parameters were used $\pi=2000, \beta=0.3, \omega_{1}=0.3, \mu=0.02, \delta_{D}=0.937, \delta_{U}=0.937$, $\kappa=0.2, \gamma=0.1, \varepsilon=0.5, \eta_{1}=0.02, \eta_{1}=0.02$.

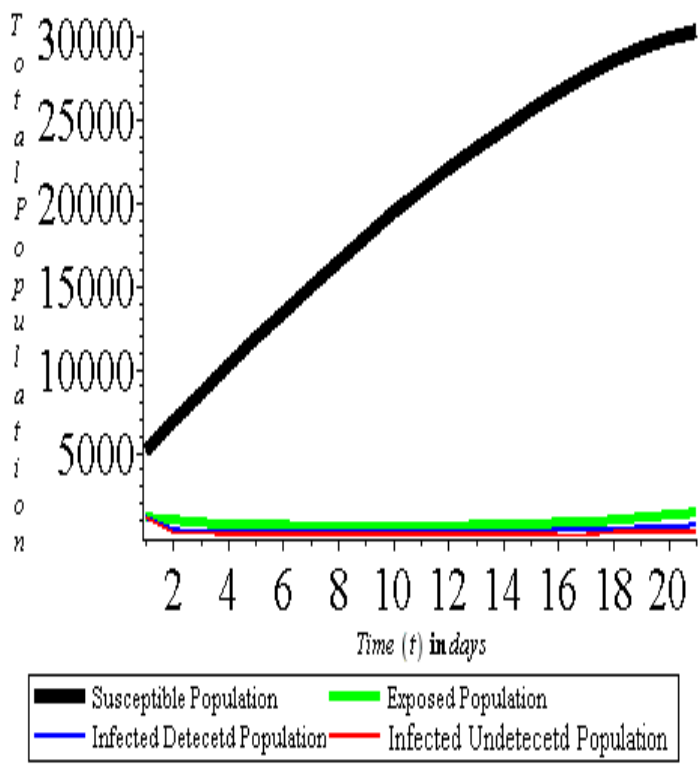

Figure 1: For the value of $\beta=0.2$

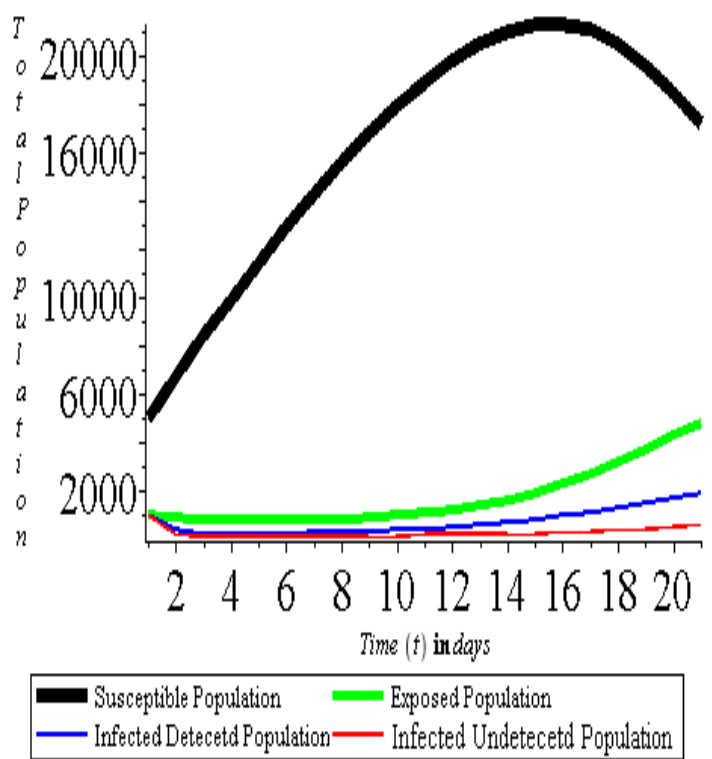

Figure 2: For the value of $\beta=0.3$ 


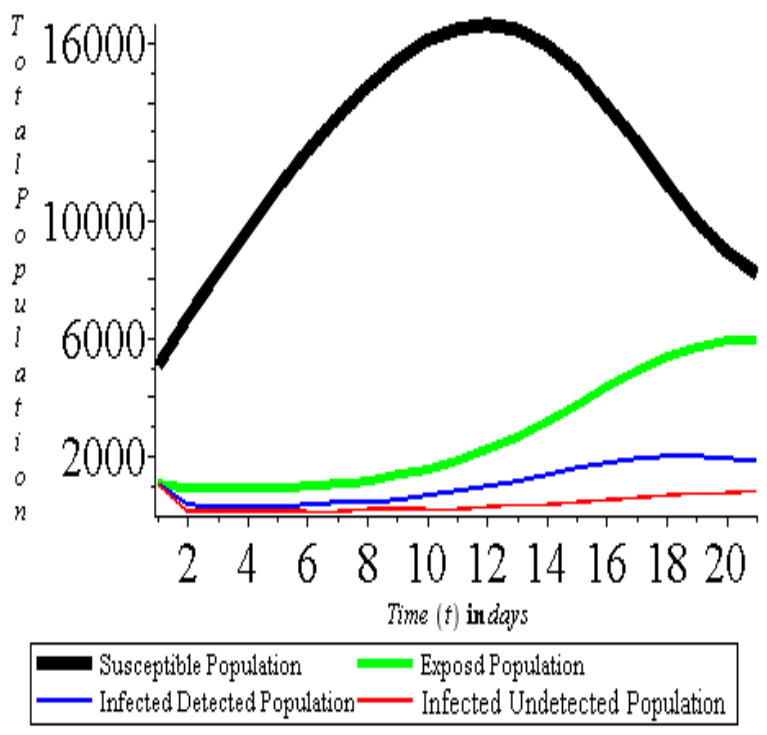

Figure 3: For the value of $\beta=0.4$
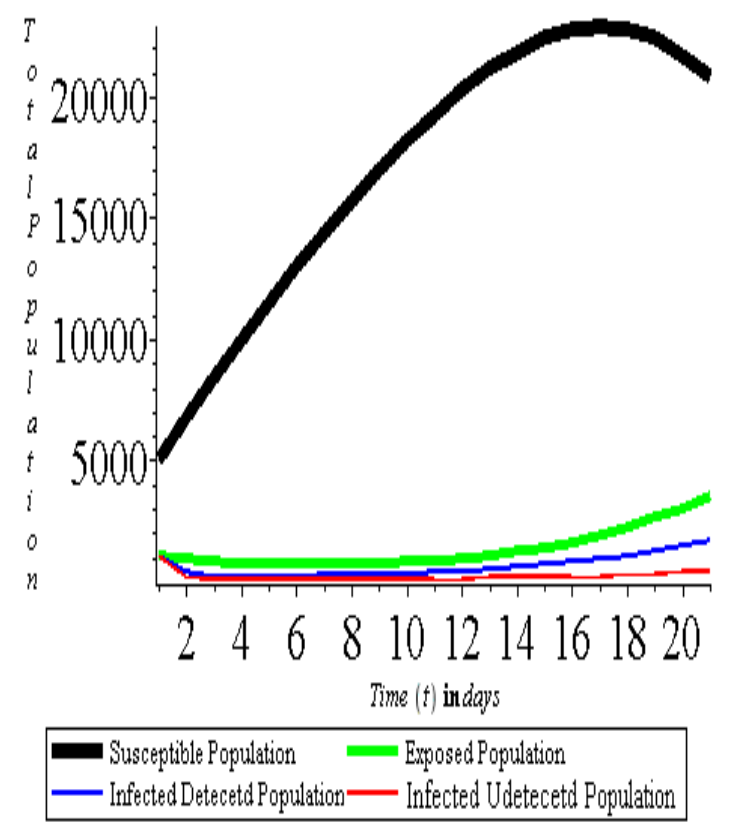

Figure 5: For the value of $\varepsilon_{1}=0.45$

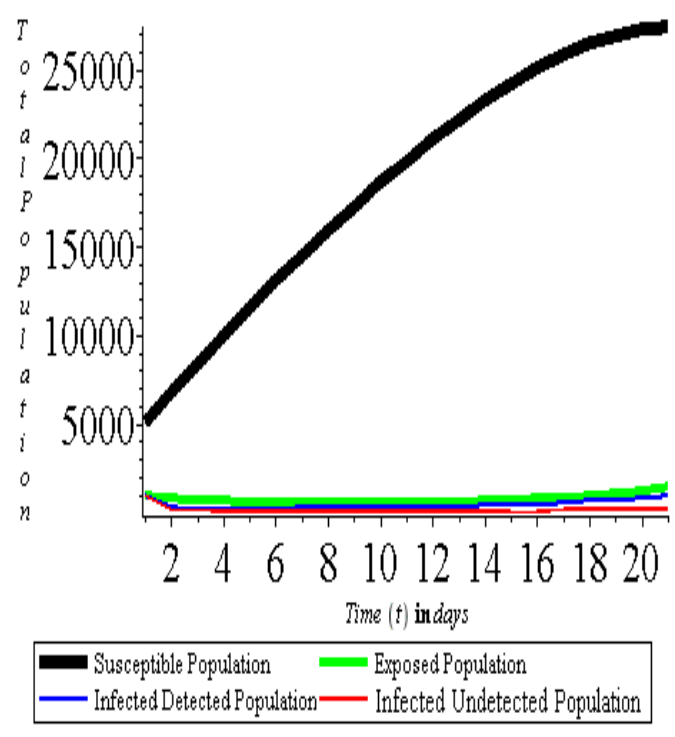

Figure 4: For the value of $\varepsilon_{1}=0.35$
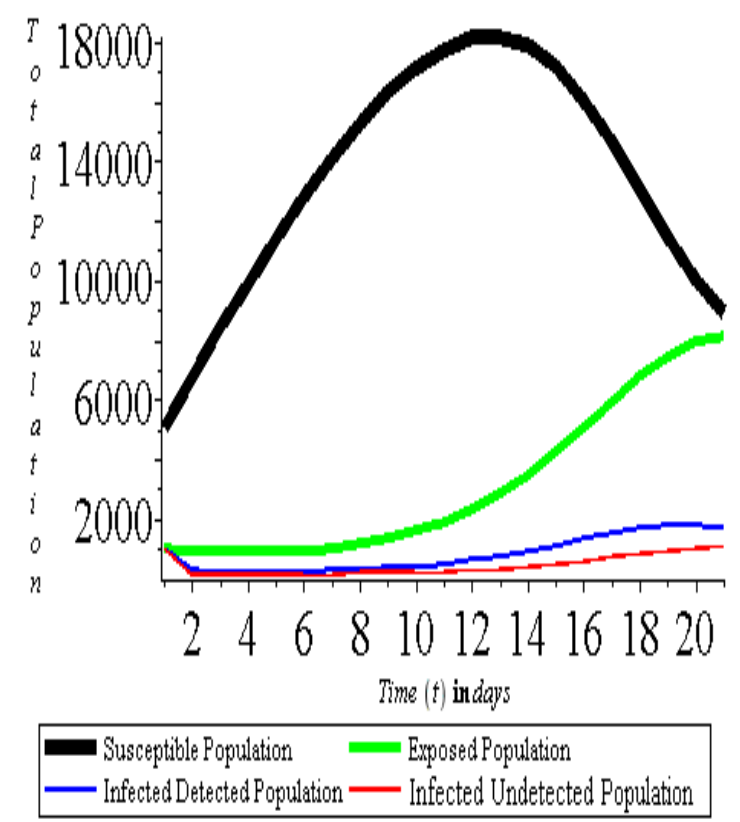

Figure 6: For the value of $\varepsilon_{1}=0.65$

\section{DISCUSSION AND CONCLUSION}

In this work, four (4) non-linear compartmental models was presented and analyzed rigorously to gain insight on the parameters that influence the outbreak of Ebola in the population. Sensitivity analysis and numerical simulations of the model were carried out to determine the effects of parameters on the outbreak of Ebola disease. In figures 1-3 above, the effect of contact rate in the human population is considered and it was observed that as the contact rate increases in the human population, the susceptible population decreases while the exposed and infected 
population increases. Also figures 4-6 shows the effect of low immunity rate in an individual which reduces the susceptible population. As the immunity in an individual decreases, it makes the exposed and infected individuals in the population to increase.

From our result, it was observed that the effective contact rate and the low immunity rate were the key parameters that influenced the dynamical spread of Ebola in the population. In conclusion, efforts should be put in place by health policy makers to reduce the rate at which an individual come in contact with the Ebola virus and also work on the immunity of an individual in order to have disease free environment.

\section{REFERENCES}

[1] Adewale S.O., Olopade I.A., Adeniran G.A., Mohammed I.T and Ajao S.O. (2015): Mathematical Analysis of effects of isolation on Ebola transmission Dynamics. Resarchjournalis. Journal of Mathematics Vol. 2|No 2 February|2015 ISSN2349-5375

[2] Amira R. and Delfin F.M. (2015): Mathematical Modeling, Simulation and Optimal Control of the 2015 Ebola outbreak in West Africa. Discrete Dynamics in Nature and society volume 2015(2015), Article ID 842792, 9 pages. http://dx.doi.org/10.1155/2015/842792

[3] Breman J.G., Johnson K.M., Van der Groen, G. (1999): A search for Ebola Virus in animals in the Democratic Replubic of the congo and Cameroon: Ecologic, vivologic, and serologic surveys 1979-1980. J.Inf.Dis.179, S139-S147.

[4] Biek R., Walsh P.D., Leroy E.M., and Real L.A. (2006): Recent Common ancesting of bola Outbreak killed 5000 gorillas. Science Pathogens 2:e90.

[5] Chowell G. and Hengartner N.W. The basic reproduction number of Ebola and the effects of public health measures: the cases of Congo and Uganda J. of theoretical Biology ELSIVIER. Available www.sciencedirect.com.

[6] Centers for Disease Control and Prevention (CDC) (2002): Ebola hermorrhagic fever information packet. Available: http://www.dcd.gov/ncidod/dvrd/spb/mnpages/fact sheets/Ebola_Fact_Booklet.pdf [accessed January 10, 2006]

[7] Centers for Disease Control (CDC) (2003b): Ebola hemorrhagic fever: table showing known cases and outbreaks, in chronological order. (http://www.dcd.gov/ncidod/dvrd/spb/mnpages/dispages/ebotabl.html), accessed on August 24, 2003.

[8] Center for Disease Control and Prevention (2016): Available at www.dcd.gov/vhflebola/about.html. [Accessed October 7, 2016

[9] Driessche P. and Watmough J. (2002): Reproduction numbers and sub-threshold endemicequilibrium for compartmental models of disease transmission, Mathematical Bi oscience 180, 29-48.

[10] Leroy E. M, Kumulungui B, Pourut X, Rouquet P, Hassanin A, and Yaba P. (2005): Fruit bats as reservoirs of Ebola Virus. Nature 438:575-576.

[11] Leirs H., Mills J.N. and Kerbs J.W. (1999): Search for the Ebola virus reservoir in Kikuit, Democratic Republic of the Congo: reflections on a vertebrate collection. J. Inf. Dis.179, S155-S163.

[12] Lakshmkanthan V., Lela S. and Martynyut A.A. (1989): Stability analysis of Non-Linear System. Marcel Dekker, Inc., New York and Basel. 
[13] Rouquet P, Fromen J, Bermejo M, kilboure A, Karesh W, and Reed P, (2005): Wild animal mortality monitoring and human Ebola Outbreaks, Gabon and Republic of Congo, 2001-2003. Emerging Infectious Diseases 11:283-290.

[14] World Health Organization (WHO), 2003a. Ebola hemorrhagic fever: disease outbreaks. (http://www.who/int/disease-outbreak-news/disease/A98.4.html)[Accessed October 17, 2003]

[15] World Health Organization (2016): Ebola virus disease Fact sheet. Available at www.who.int/mediacentre/factsheets/fs 103/en/. [Accessed October 9, 2016]

[16] World Health Organization (WHO), 2003b. Ebola hemorrhagic fever. (http://www.who.int/inf-fs/en/fact103.html), [Accessed August 24, 2003]

[17] Zack Yarus (2012): A mathematical look at Ebola Virus. May 11, 2012. 